Case Report

\title{
Patient with Marfan Syndrome and a Novel Variant in FBN1 Presenting with Bilateral Popliteal Artery Aneurysm
}

\author{
Ahmed Mohammad, ${ }^{1,2}$ Haytham Helmi, ${ }^{1,2}$ and Paldeep S. Atwal (iD) \\ ${ }^{1}$ Department of Clinical Genomics, Mayo Clinic, Jacksonville, FL, USA \\ ${ }^{2}$ Center for Individualized Medicine, Mayo Clinic, Jacksonville, FL, USA \\ Correspondence should be addressed to Paldeep S. Atwal; paldeep.atwal@gmail.com
}

Received 1 November 2017; Accepted 2 January 2018; Published 29 March 2018

Academic Editor: Philip D. Cotter

Copyright ( $) 2018$ Ahmed Mohammad et al. This is an open access article distributed under the Creative Commons Attribution License, which permits unrestricted use, distribution, and reproduction in any medium, provided the original work is properly cited.

We present a 43-year-old man with aortic root dilation, mitral valve prolapse, and marfanoid appearance, who presented with acute onset left leg pain. He underwent a Doppler ultrasound that revealed left popliteal artery aneurysm with thrombus. CT angiogram showed bilateral popliteal artery aneurysms. After repairing of his left popliteal artery aneurysm, he was sent for genetic evaluation. He was diagnosed with Marfan syndrome (MFS) based on the revised Ghent criteria and then underwent FBN1 sequencing and deletion/duplication analysis, which detected a novel pathogenic variant in gene FBN1, denoted by c.5872 T>A (p.Cys1958Ser). MFS is a connective tissue disorder with an autosomal dominant inheritance due to pathogenic variants in FBN1 that encodes Fibrillin1, a major element of the extracellular matrix, and connective tissue throughout the body. MFS involves multiple systems, most commonly the cardiovascular, musculoskeletal, and visual systems. In our case we present a rare finding of bilateral popliteal artery aneurysms in a male patient with MFS.

\section{Background}

MFS is a genetic connective tissue disorder with an autosomal dominant inheritance that involves multiple systems, most commonly the cardiovascular, musculoskeletal, and ocular systems [1]. It is caused by pathogenic variants in FBN1 that encodes Fibrillin-1, a major element of the extracellular matrix, and connective tissue throughout the body [2]. Due to the improved survival and longer life expectancy of patients with MFS, less common phenotypic features continue to be observed [3]. MFS is diagnosed based on Ghent criteria, which takes into account clinical manifestations, genetic testing, and family history [4]. In this report we present a male patient with clinical features of MFS, a novel pathogenic variant in $F B N 1$, and the unusual finding of bilateral popliteal aneurysms.

\section{Case Presentation}

Our proband is a 43-year-old male of northern European descent who initially presented with acute onset left leg cramping pain that lasted for several days. The pain was associated with numbness in his left leg and nausea. His past medical history was significant for mitral valve prolapse, aortic root dilation umbilical hernia left anterior cruciate ligament (ACL) rupture, and severe myopia corrected with LASIK surgery. Family history revealed rheumatic heart disease in his mother, necessitating mitral valve repair due to mitral valve prolapse with severe regurgitation. Upon examination, his popliteal arterial pulses were 4+ bilateral, bounding. His height was $196 \mathrm{~cm}$, with an increased arm span-height ratio of 1.07. He had a mild pectus carinatum deformity, mild scoliosis positive wrist and thumb sign, pes planus, and mild skin striae. A Doppler ultrasound was performed by his primary care physician (PCP), which ruled out deep venous thrombosis (DVT); however, it revealed a large left popliteal artery aneurysm with thrombus that measured $6.3 \times 3.1 \times 3.4 \mathrm{~cm}$. He underwent a CT angiogram that showed normal abdominal aorta as well as common iliac arteries. However it showed bilateral popliteal artery aneurysms measuring $3.6 \times 4 \mathrm{~cm}$ in the left side and $3 \times$ $2.8 \mathrm{~cm}$ in the right side (Figure 3). After repairing of his left 
TABLE 1

\begin{tabular}{lc}
\hline Feature & Value \\
\hline Wrist and thumb sign & 3 \\
Pectus carinatum deformity & 2 \\
Hindfoot deformity & 2 \\
Plain flat foot & 1 \\
Scoliosis & 1 \\
Skin striae & 1 \\
Severe myopia & 1 \\
Mitral valve prolapse & 1 \\
Total & 12 \\
\hline
\end{tabular}

popliteal artery aneurysm with bovine carotid interposition via posterior approach, he was sent for cardiac and genetic evaluations. He underwent a MRI exam of the heart with and without intravenous gadolinium contrast administration (Figure 2) which showed mild bileaflet mitral valve prolapse and aortic root dilation (measuring $46 \mathrm{~mm}$ at the level of the sinuses of Valsalva). The patient has been prescribed metoprolol tartrate and losartan to control his aortic root disease and was to undergo elective aortic repair when the aortic dimension becomes near to or reaches $50 \mathrm{~mm}$. A diagnosis of Marfan syndrome was made based on the revised Ghent criteria as the proband had aortic root dilation $(Z$ score: 3.72 ) and a systemic score of 12 (Table 1). His diagnosis was further confirmed after undergoing FBN1 sequencing and deletion/duplication analysis, which detected a novel pathogenic variant in gene $F B N 1$, denoted by c.5872 T>A (p.Cys1958Ser) (Figure 1).

\section{Discussion}

Arterial aneurysms are most commonly caused by atherosclerotic disease, especially in elderly patients aged over 60 . Other etiologies, such as connective tissue disorders, should be investigated in younger patients such as ours $[5,6]$. Fibrillin-1 microfibrils, through interactions with elastin and other proteins, provide structure to elastic and nonelastic connective tissues $[7,8]$. In addition to the architectural functions, Fibrillin-1 plays an important role in regulating TGF$\beta$ complexes in the extracellular matrix. TGF- $\beta$ signaling controls various processes at the cellular level, such as cellular growth, differentiation, and apoptosis $[9,10]$. When Fibrillin1 is defective, it disrupts the normal architecture of connective tissues. Elastic fibers, found in tissues such as aortic media, become disorganized and fragmented resulting in the loss of elastic properties which causes the vessels to be less compliant and more prone to dilation and aneurysm formation [10,11].

Pathogenic variants are identified in $72-93 \%$ of individuals fulfilling a clinical diagnosis of MFS based on Ghent nosology $[12,13]$. The likelihood of detecting a pathogenic variant decreases for those individuals not meeting Ghent criteria [12]. Large intragenic deletions in the FBN1 gene have been detected in approximately $2 \%$ of individuals with MFS who did not have a pathogenic variant identified by sequence analysis [14].

In our case a pathogenic variant has been identified in the FBN1 gene. The C1958S variant has not been published as pathogenic or been reported as benign to our knowledge. The C1958S variant is a nonconservative amino acid substitution, which is likely to impact secondary protein structure as these residues differ in polarity, charge, and size. This substitution occurs at a position that is conserved across species and in silico analysis predicts this variant is probably damaging to the protein structure or function. Additionally, C1958S affects a Cysteine residue within a calcium-binding EGF-like domain of the FBN1 gene, which may affect disulfide bonding and is predicted to alter the structure and functions of the protein. Cysteine substitutions in the calcium-binding EGFlike domains represent the majority of pathogenic missense changes associated with FBN1-related disorders [2]. Furthermore, other missense variants at the same residue (C1958Y, C1958R) have also been reported in published literature in association with FBN1-related disorders, including assumed de novo occurrences for both variants, supporting the functional significance of this residue [15-17]. Finally, this variant has not been observed in large population cohorts such as the ExAC (Exome Aggregation Consortium) database [18-20]. Based on these data, ACMG variant classification guidelines [21] classify our variant as pathogenic (IIIa) (evidence: PM2, PS1, PM5, PP3, BS4, and PM1).

Peripheral vascular aneurysms in patients with Marfan syndrome have been reported on rare occasions [5, 22-24]. Bilateral popliteal aneurysms in MFS have been described on two occasions before $[5,23]$, but in one of these studies, it was believed that autosomal dominant polycystic kidney disease (ADPKD) was the main contributor to this manifestation [5]. In our case the patient did not have any other medical conditions besides his MFS diagnosis. Popliteal aneurysms should be properly investigated and repaired, as they can result in serious complications such as thrombus formation or rupture.

Based on history, the mother's mitral valve prolapse with severe regurgitation was attributed to rheumatic valve disease rather than a genetic connective tissue disorder. However, it is still a very likely possibility as her son has a confirmed diagnosis of MFS. She is yet to undergo the FBN1 sequencing.

Due to its rarity in MRS we do not recommend screening for peripheral vascular aneurysms in patients diagnosed with MRS as we do not think it is a cost-effective approach. However, we do recommend that patients with popliteal or other peripheral aneurysms, especially those who are relatively young and without risk factors, should undergo further clinical and genetic assessment in order to confirm/exclude the possibility of inherited connective tissue disorders.

In summary we describe the case of a 43-year-old male with a novel pathogenic variant in FBN1 causing Marfan syndrome with the rare phenotype of bilateral popliteal aneurysms. Further advancements in medical genetics will result in continued expansion and discovery of phenotypes as described above.

\section{Abbreviations}

MFS: Marfan syndrome

ACL: Anterior cruciate ligament

ADPKD: Autosomal dominant polycystic kidney disease. 


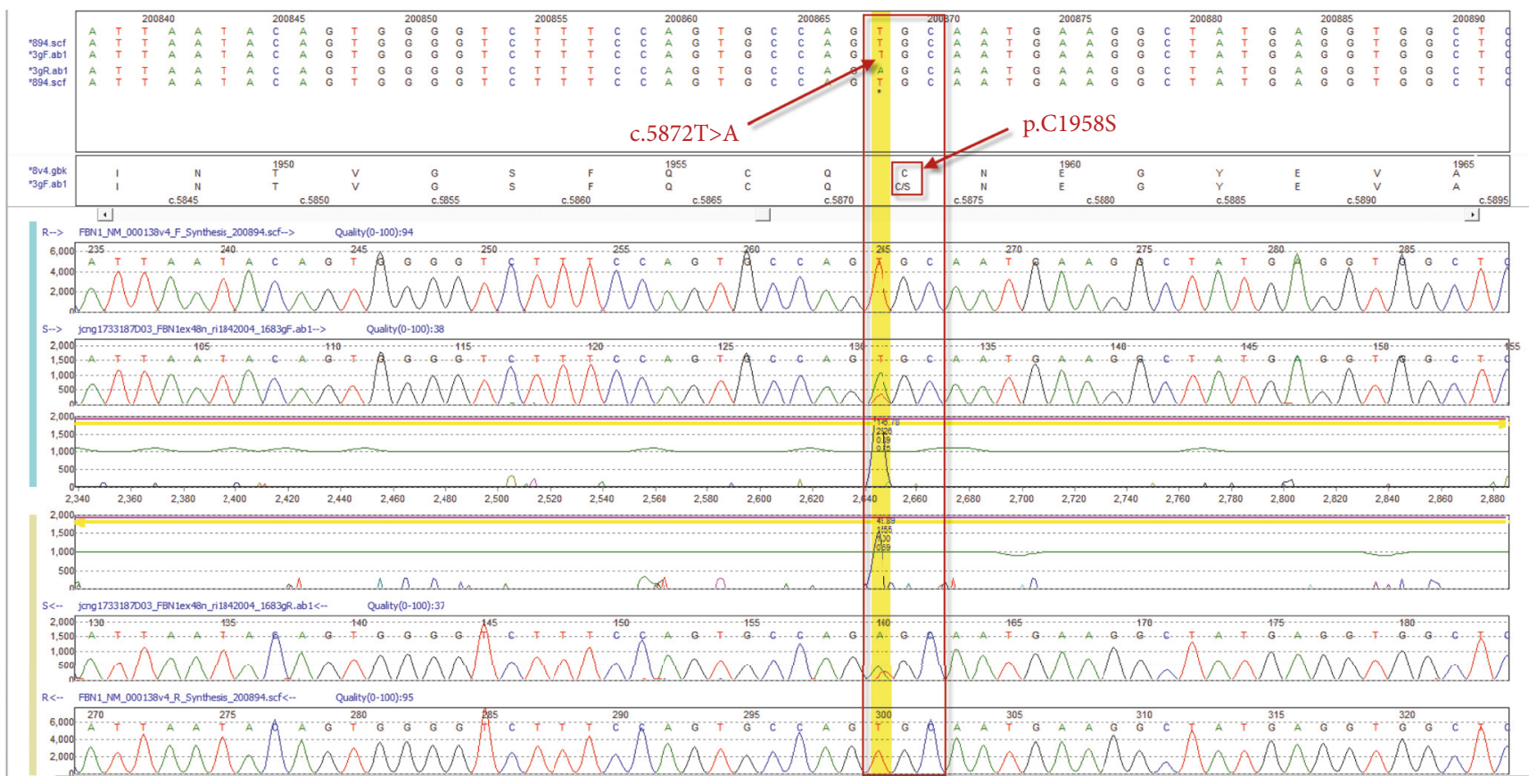

FIGURE 1: DNA chromatogram demonstrating heterozygous c.5872T >A variant in FBN1.

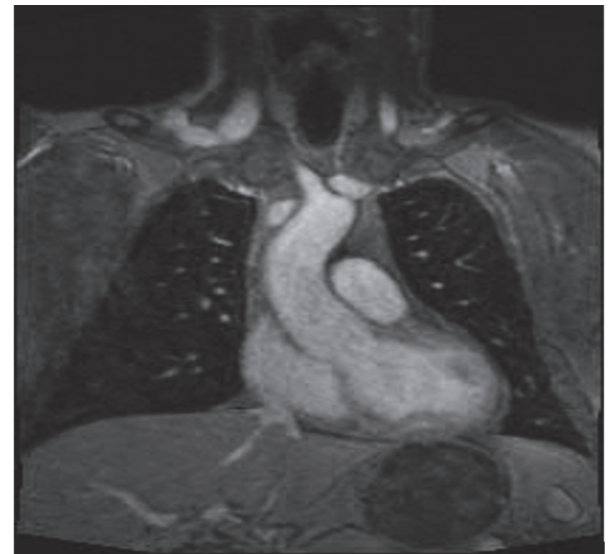

FIGURE 2: MRI of the heart with and without intravenous gadolinium contrast administration.

\section{Ethical Approval}

All procedures followed were in accordance with the ethical standards of the responsible committee on human experimentation (institutional and national) and with the Helsinki Declarations of 1975, as revised in 2000 (5).

\section{Consent}

Informed consent was obtained from all patients for being included in the study.

\section{Conflicts of Interest}

All authors declare that there are no conflicts of interest.

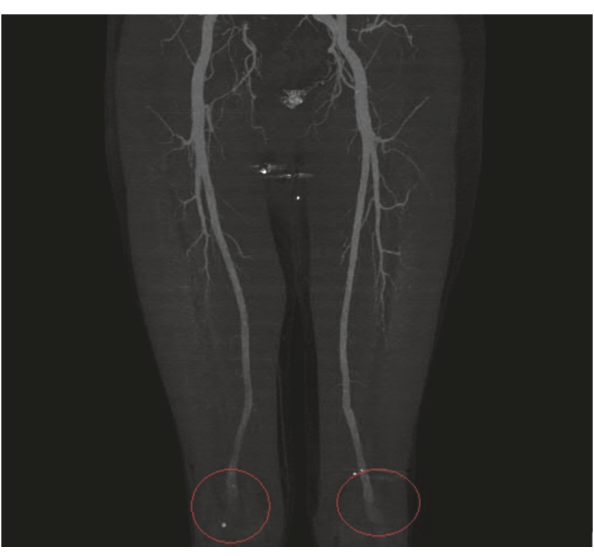

FIGURE 3: CT angiogram MIP images showing bilateral popliteal aneurysms highlighted by red circles.

\section{Authors' Contributions}

All authors made substantial contributions to the conception or design of the work or the acquisition, analysis, or interpretation of data for the work, drafting of the work, or revising it critically for important intellectual content, gave final approval of the version to be published, and agreed to be accountable for all aspects of the work in ensuring that questions related to the accuracy or integrity of any part of the work are appropriately investigated and resolved.

\section{Acknowledgments}

The Center for Individualized Medicine, Mayo Clinic, provided funds to support investigator time. 


\section{References}

[1] T. Takayama, T. Miyata, and H. Nagawa, "True abdominal aortic aneurysm in Marfan syndrome," Journal of Vascular Surgery, vol. 49, no. 5, pp. 1162-1165, 2009.

[2] G. Collod-Béroud, S. Le Bourdelles, L. Ades et al., "Update of the UMD-FBN1 mutation database and creation of an FBN1 polymorphism database," Human Mutation, vol. 22, no. 3, pp. 199-208, 2003.

[3] R. E. Pyeritz, "Recent progress in understanding the natural and clinical histories of the Marfan syndrome," Trends in Cardiovascular Medicine, vol. 26, no. 5, pp. 423-428, 2016.

[4] B. L. Loeys, H. C. Dietz, A. C. Braverman et al., "The revised Ghent nosology for the Marfan syndrome," Journal of Medical Genetics, vol. 47, no. 7, pp. 476-485, 2010.

[5] W. Al-Hakim and D. J. A. Goldsmith, "Bilateral popliteal aneurysms complicating adult polycystic kidney disease in a patient with a marfanoid habitus," Postgraduate Medical Journal, vol. 79, no. 934, pp. 474-475, 2003.

[6] T. Trajbar, "A rare case of multiple aneurysms in a young patient," Collegium Antropologicum, vol. 30, no. 1, p. 235, 2006.

[7] H. C. Dietz and R. E. Pyeritz, "Mutations in the human gene for fibrillin-1 (FBN1) in the Marfan syndrome and related disorders," Human Molecular Genetics, vol. 4, pp. 1799-1809, 1995.

[8] L. Pereira, K. Andrikopoulos, J. Tian et al., "Targetting of the gene encoding fibrillin-1 recapitulates the vascular aspect of marfan syndrome," Nature Genetics, vol. 17, no. 2, pp. 218-218, 1997.

[9] Z. Isogai, R. N. Ono, and S. Ushiro, "Latent transforming growth factor $\beta$-binding protein 1 interacts with fibrillin and is a microfibril-associated protein," The Journal of Biological Chemistry, vol. 278, no. 4, pp. 2750-2757, 2003.

[10] A. W. Y. Chung, K. Au Yeung, G. G. S. Sandor, D. P. Judge, H. C. Dietz, and C. Van Breemen, "Loss of elastic fiber integrity and reduction of vascular smooth muscle contraction resulting from the upregulated activities of matrix metalloproteinase-2 and -9 in the thoracic aortic aneurysm in Marfan syndrome," Circulation Research, vol. 101, no. 5, pp. 512-522, 2007.

[11] N. M. Ammash, T. M. Sundt, and H. M. Connolly, "Marfan syndrome-diagnosis and management," Current Problems in Cardiology, vol. 33, no. 1, pp. 7-39, 2008.

[12] C. Stheneur, G. Collod-Béroud, L. Faivre et al., "Identification of the minimal combination of clinical features in probands for efficient mutation detection in the FBN1 gene," European Journal of Human Genetics, vol. 17, no. 9, pp. 1121-1128, 2009.

[13] C. Boileau, G. Jondeau, T. Mizuguchi, and N. Matsumoto, "Molecular genetics of Marfan syndrome," Current Opinion in Cardiology, vol. 20, no. 3, pp. 194-200, 2005.

[14] G. Mátyás, S. Alonso, A. Patrignani et al., "Large genomic fibrillin-1 (FBN1) gene deletions provide evidence for true haploinsufficiency in Marfan syndrome," Human Genetics, vol. 122, no. 1, pp. 23-32, 2007.

[15] N. Ogawa, Y. Imai, Y. Takahashi et al., "Evaluating Japanese patients with the Marfan syndrome using high-throughput microarray-based mutational analysis of fibrillin-1 gene," American Journal of Cardiology, vol. 108, no. 12, pp. 1801-1807, 2011.

[16] C. A. Valencia and et al., "Clinical Impact and CostEffectiveness of Whole Exome Sequencing as a Diagnostic Tool: A Pediatric Center's Experience," Front Pediatr, vol. 3, p. 67, 2015.
[17] H. Yang, M. Luo, Y. Fu et al., "Genetic testing of 248 Chinese aortopathy patients using a panel assay," Scientific Reports, vol. 6, Article ID 33002, 2016.

[18] M. Lek, K. J. Karczewski, and E. V. Minikel, "Analysis of proteincoding genetic variation in 60,706 humans," Nature, vol. 536, no. 7616, pp. 285-291, 2016.

[19] G. R. Abecasis, A. Auton, L. D. Brooks et al., "An integrated map of genetic variation from 1,092 human genomes," Nature, vol. 491, pp. 56-65, 2012.

[20] Exome Variant Server, “N.G.E.S.P.E., Seattle, WA," http://evs.gs .washington.edu/EVS/.

[21] S. Richards, N. Aziz, and S. Bale, "Standards and guidelines for the interpretation of sequence variants: a joint consensus recommendation of the American College of Medical Genetics and Genomics and the Association for Molecular Pathology," Genetics in Medicine, vol. 17, no. 5, pp. 405-423, 2015.

[22] K. Morisaki, M. Kobayashi, H. Miyachi et al., "Subclavian artery aneurysm in Marfan syndrome," Annals of Vascular Surgery, vol. 26, no. 5, pp. 731-e4, 2012.

[23] Y. Tijani and et al., "Exceptional association of bilateral popliteal aneurysm with an abdominal aortic aneurysm in Marfan syndrome," Journal Des Maladies Vasculaires, vol. 39, no. 4, pp. 278-281, 2014.

[24] B. Wolfgarten, I. KrÃijger, and M. Gawenda, "Rare Manifestation of Abdominal Aortic Aneurysm and Popliteal Aneurysm in a Patient with Marfan's Syndrome: A Case Report," Vascular and Endovascular Surgery, vol. 35, no. 1, pp. 81-84, 2001. 


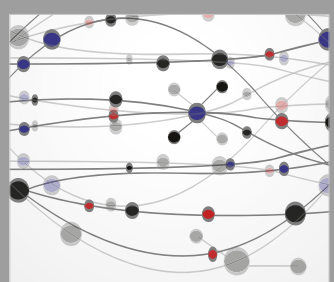

The Scientific World Journal
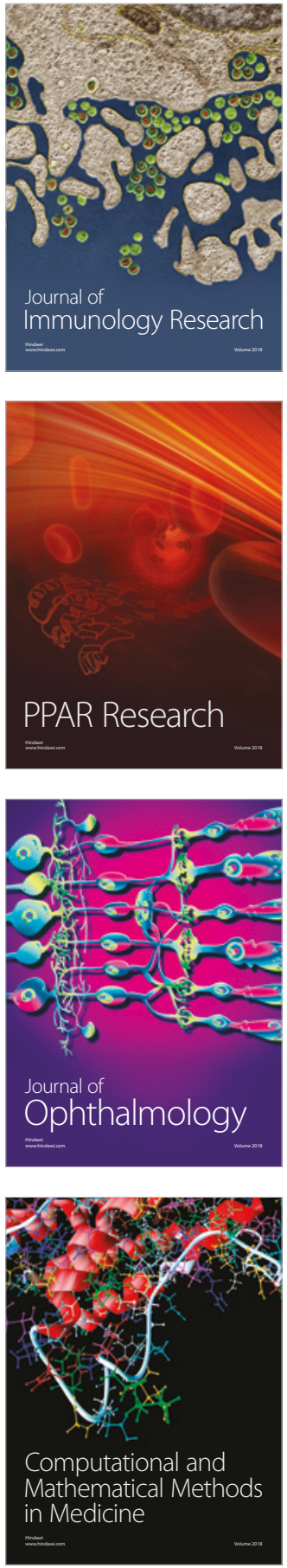

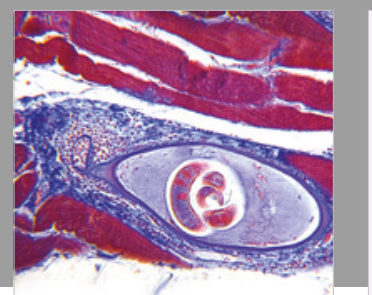

Gastroenterology Research and Practice

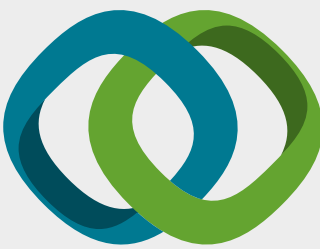

\section{Hindawi}

Submit your manuscripts at

www.hindawi.com
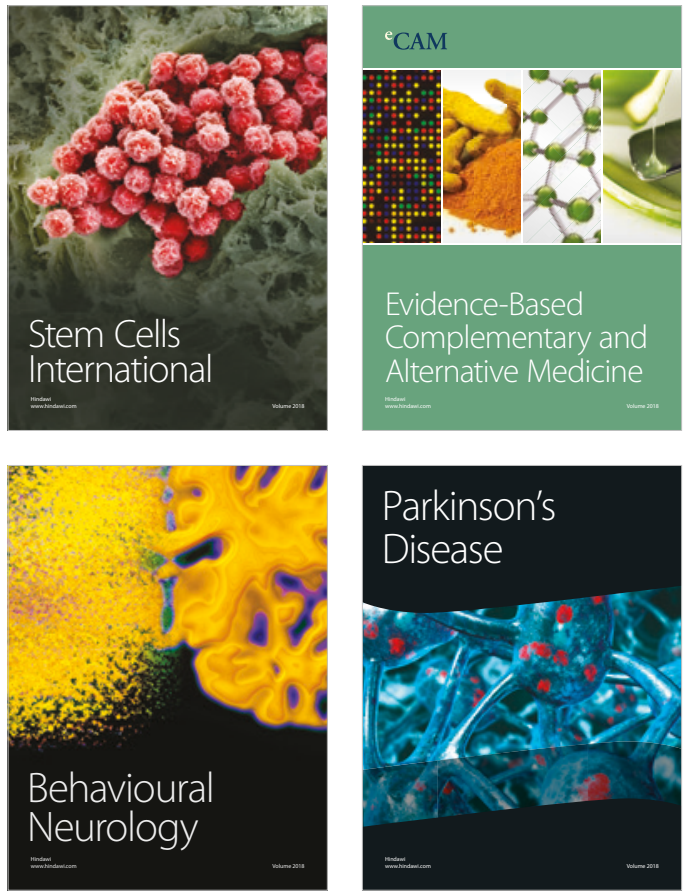

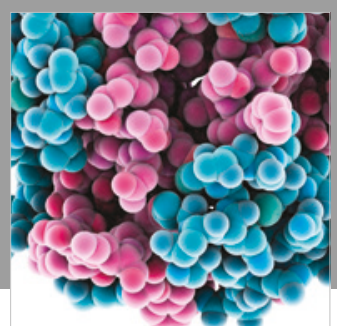

ournal of

Diabetes Research

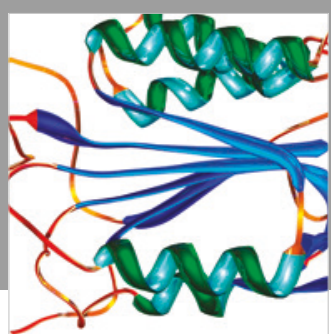

Disease Markers
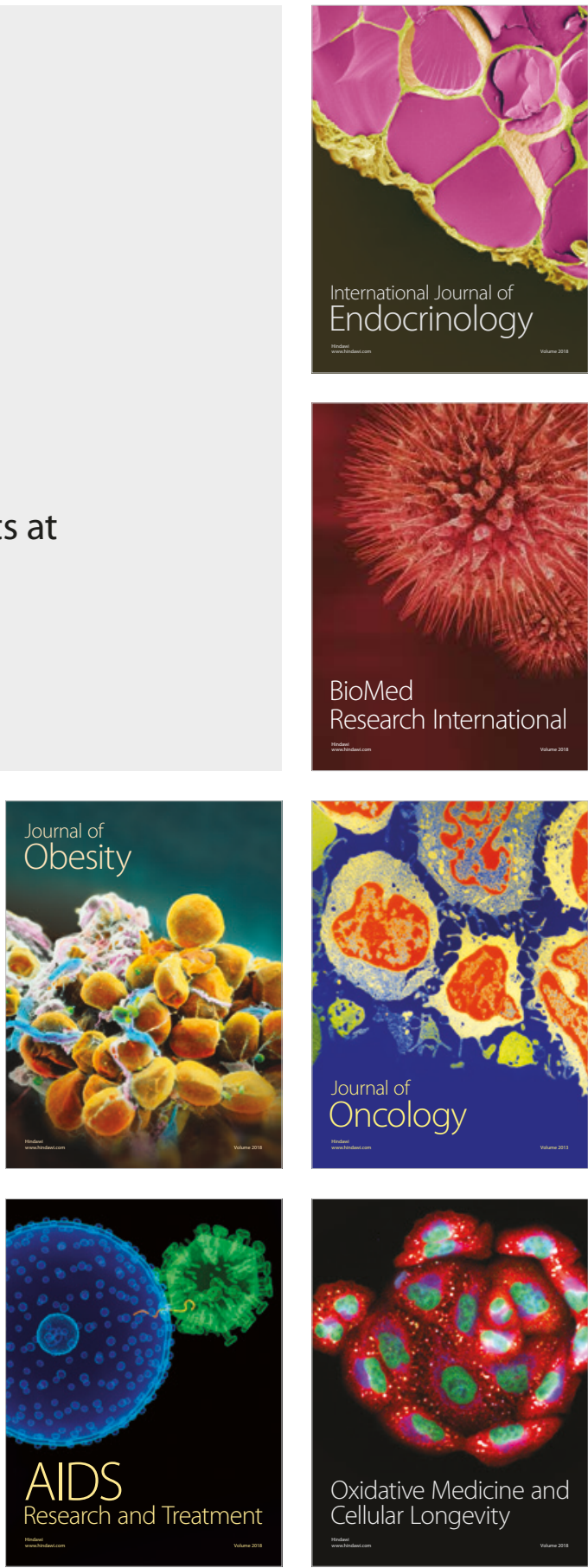